\title{
Alpha-rhythm stimulation using brain entrainment enhances heart rate variability in subjects with reduced HRV
}

\author{
Francesco Casciaro ${ }^{1}$, Vincenza Laterza ${ }^{1}$, Sergio Conte ${ }^{1}$, Maria Pieralice ${ }^{1,2}$, Antonio Federici ${ }^{3}$, \\ Orlando Todarello ${ }^{4}$, Franco Orsucci ${ }^{5}$, Elio Conte ${ }^{1,3,4^{*}}$ \\ ${ }^{1}$ School of Advanced International Studies on Applied Theoretical and Non Linear Methodologies of Physics, Bari, Italy \\ ${ }^{2}$ ISPA-CNR, Bari, Italy \\ ${ }^{3}$ Department of Biomedical Sciences and Oncology, TIRES, Center of Innovative Technologies for Signal Detection and Processing, \\ University of Bari, Bari, Italy \\ ${ }^{4}$ Department of Neurological Sciences, Center of Innovative Technologies for Signal Detection and Processing, University of Bari, \\ Bari, Italy \\ ${ }^{5}$ Division of Psychology and Language Sciences University College, London, UK \\ Email: "elio.conte@fastwebnet.it
}

Received 12 June 2013; revised 8 August 2013; accepted 2 September 2013

Copyright (C 2013 Francesco Casciaro et al. This is an open access article distributed under the Creative Commons Attribution License, which permits unrestricted use, distribution, and reproduction in any medium, provided the original work is properly cited.

\begin{abstract}
In the present research, we have used the brain entrainment (BWE) treatment simultaneously recording time series data of R-R intervals of the ECG during rest condition. In detail, we have used alpha brain stimulation and we have found that it induces an enhancement of HRV, particularly in Total Variability and Vagal Modulation activities. The experiment has been performed by us on ten subjects with age ranging from 20 to 70 years old. The risk induced from low HRV is by this time well known in literature. Therefore, the obtained result promises to be of valuable interest not only in terms of the basic neurological investigation but also because it delineates new possibilities in terms of clinical application.
\end{abstract}

Keywords: Brain Entrainment; Alpha Rhythm Brain Stimulation; HRV Analysis; Fast Fourier Transform Analysis of Time Series Data

\section{INTRODUCTION}

It is well known that brainwave entrainment is a process that synchronizes or entrains natural brainwaves to that of an external stimulus frequency, which is usually auditory and/or visual in nature. Brainwave entrainment (BWE) is a well-known physical technique relating using rhythmic stimuli that induce a frequency response of brainwaves to match the frequency of the stimulus. This method is evidencing its importance in some specific fields.

\footnotetext{
"Corresponding author.
}

We give here only some indications as to improve cognitive functioning, mood, stress/anxiety, pain relief, headaches/migraines, behaviour, and premenstrual syndrome (PMS). The basic theoretical foundation in BWE is to stimulate the brain at the desired frequency via auditory tones, flashing lights, or a combination of both using isochronic, monaural, or binaural beats. Isochronic tones are evenly spaced tones that simply turn on and off. Monaural and Binaural beats are presented as two tones with very similar frequencies, and obviously the brain perceives a beat that is the difference between the two pitches. The pitches are presented together with monaural beats but fed separately to each ear with binaural beats.

For photic stimulation, most devices use lights or a flashing screen. Pulses of light can be presented as different waveforms or colours. Photic stimulation can be also presented independently to each eye or each visual field in order to more effectively target stimulation to the right or left brain hemisphere.

Under a rather phenomenological profile BWE has an old story. In fact, the first known clinical application of BWE was introduced from the French psychologist, Pierre Janet, in the late 1800s. After the initial demonstration of Berger in 1934 on electrical activity recorded from the human brain, Adrian and Mathews showed that the Berger rhythm (alpha) could be further amplified by photic stimulation at the same frequency. In 1942, Dempsey and Morison found that BWE could also be induced by a tactile stimulus, and in 1959 Chaitran reported entrainment effects with an auditory stimulus. Psychological effects of BWE were further explored in 1946 when flickering light produced frequency-depen- 
dent sensations of pattern, movement and colour. The development of BWE tools increased largely starting with 1973. Oster's results evidenced the properties of the binaural beat. Research on the effects of BWE on pain, headaches, migraines, anxiety, and stress followed in the 1980s and expanded in the 1990s to include learning and memory, ADHD, learning disabilities, behavioural problems, and PMS.

The presently technological level reached in computer assisted imaging elaboration as well as in the use of new methodologies of analysis has induced valuable new interest in the theoretical analysis of the treatment as well as in the clinical applications. An excellent review and an historical account of the first authors and recent advances in this field are given in [1].

It should rather complex to elucidate in this paper all the potentialities of the BWE. Therefore we limit ourselves to the result that relates directly our present paper. It results that the presentation of a consistent rhythmic stimulus within 8 to $10 \mathrm{~Hz}$ causes brainwaves in the occipital lobe, parietal lobe, or temporal cortex to exhibit a frequency-following response that either resonates with the presenting stimulus or shows a frequency harmonic or a sub-harmonic of a stimulus.

The finality of the present contribution is to give support to clinicians and researchers as BWE therapeutic tool, in a new field, the Heart Rate variability.

We have articulated our research on the basis of the following indications.

It is known that the section of the nervous system which controls the visceral functions of the body is the autonomous nervous system (ANS) that regulates itself. This system tonically and reflexively influences blood pressure, peripheral resistance, cardiac frequency and cardiac debt. In order to reach it, it receives autonomic influence. One of its main characteristics consists of constant alteration of its beating frequency. The information reaches the central nervous system (nucleus tractus solitarius) through marrow and vagal afferent ways; is modulated and returns to the heart through fast vagal efferent fibers and slow sympathetic efferent ones. The resulting effect of these autonomic influences is the variability beat after beat of the instantaneous heart rate.

The key concept here in physiological terms is that one of Variability. The physiological importance of such concept of Variability has been discussed by us in detail in some previous papers where we have given detailed methods for its investigation and quantification [2-5]. The heart receives information from the amygdala. In turn the amygdala receives it from the thalamus.

The quality of incoming information modifies the Heart Rate Variability (HRV) that is then transmitted through the body neurologically (back to the amygdala, the brain stem, and to higher cortical centres), hormonally, and biophysically. HRV in heart acts as an extension and amplifier of the limbic system of the body. It contains its own perception of an event.

We may anticipate here that HRV has reached recently the greatest consideration since, without any doubt, it represents the most complex and, on the other hand, the most important marker under the physiological, psychophysiological and also neurological profile. In 1977, Wolf et al. were the first ones to demonstrate association between increase in the risk for post-infarct mortality with decreased Heart Rate Variability (HRV). Actually, the capacity to vary the cardiac beat rate has the physiological meaning of adapting the Cardiovascular System to the several daily situations, from sleep to strenuous physical activity and strong cognitive or emotional engagement. The study of the HRV has allowed recognizing and characterizing some situations in which diseases affect the autonomic control. Some authors have demonstrated that the decrease of the HRV is related to a higher index of cardiovascular morbidity and mortality implying the presence of individual physiological dysfunction. For a detailed discussion see [5].

A healthy physiological system has the following characteristics: efficient neural control, rhythmic physiological variability within normal limits, greater responsepotential to challenge, greater range of response behaviour. The basic importance of the concept of Variability is represented from the fact that an attenuated physiological variability is associated with a lack of psychological and behavioural flexibility in response to environmental demand. A reduction in HRV is therefore not only an indication of a lack of physiological variability, but also in its broad sense a reflection of reduced psychological and behavioural flexibility.

Living systems are open systems far from thermodynamic equilibrium. This is to say that they are flexible systems that constantly exchange matter and energy with their background. Hear Rate does not escape to this mechanism and, in particular, it is called to constantly respond to the demand of changing its intrinsic modulation as answer to the continuous requirement arising form the inner or the outside. Such flexibility in living organisms is reached by the presence of so called non linearity that assures to the considered system to realize self-organization, complexity in its dynamics and great modulation and flexibility as response to the continuous changing physiological requirements. The heart beat rate is a non linear, complex, non stationary and non periodic signal $[2,3]$ subjected to fluctuations. The ideal fluctuation is a quite rhythmic increase and decrease in HR which generates a continuous variability in time of the signal. Actually, as previously said, HRV behaviour is 
typically a largely complex pattern [2-6]. The nature of the heart rate variability describes the particular rhythm of an individual heart beat characteristics, and this is related to a number of effects regarding other body systems. All other oscillating systems function, in fact, in accordance with the coherence or otherwise of the heart beat variability. Continuous studies in the last ten years have explained that heart rate variability must be contained within some values for normal subject behaviour. Reduced variability is the first sign of increased subject risk of sudden heart failure [5,6]. Still, growing evidence suggests that alterations in autonomic function are related as example to the pathophysiology of panic disorder (PD). The risk of adverse clinical cardiac events is increased in patients with PD [5].

Autonomic nervous system (ANS) dysfunction and reduced heart rate variability (HRV) have been reported in a wide variety of psychological and psychiatric disorders as, in particular, anxiety and stress. Some authors recorded cardiac activity and assessed HRV in acutely hospitalized manic bipolar (BD) and schizophrenia (SCZ) patients. Generally speaking, autonomic dysregulation is associated with more severe psychiatric symptoms, suggesting HRV dysfunction. For an extensive discussion about the different pathologies that have been investigated we suggest the reader our recent paper [5].

In conclusion, HRV is a very important marker of autonomic activity and thus a privileged observatory for a lot of disease conditions. Some of these conditions are very elusive and practically impossible to detect with reliable diagnosis methods. Some subjects may exhibit a psychological profile with a tendency to the introversion, anxiety and depression without showing psychological test values out of the normal established values. Other subjects may have tendency to develop pathologies with a relative lowering of the threshold pain as example in the headache, migraine. All such subjects may exhibit reduced HRV and thus enhanced risk factor. This justifies the interest in the development of analysis by HRV methods, both under the profile of the diagnosis as well as of the treatment based to enhance reduced heart variability.

On this basis we attempted to experience if subjects with no defined diagnosis of anxiety and of stress or depression, not of sedentary style of life, but starting, however, with a reduced variability in HRV, when submitted to BWE treatment, reported a net improvement in HRV. Note that under the therapeutic profile we have not presently many alternatives. In addition to some pharmacological treatment, actually we have biofeedback as well as the technique of the controlled respiration. Certainly we well know the effect of the respiratory cycle on baroreflex and vague modulation. As consequence we apply this treatment that of course should require an appropriate identification of a resonance frequency in order to realize a respiration in phase with respiration and with net difference of phase in BP. Resonant frequency heart rate variability biofeedback increases baroreflex gain and peak expiratory flow [7]. The limitation of the method consists in the basic difficulty to identify the correct resonant frequency by using a linear method as the Fast Fourier Transform (FFT) and in the actual application to subjects having problems when due as example to psychological disorders that may compromise the correct training also with the direct help of an assistant operator. Finally, it relates CNS and ANS only marginally since we have not a direct but only an indirect action on the brain. Consequently, it becomes rather evident that our proposed technique, if confirmed, could represent an important alternative by clinicians and researchers of BWE as basic therapeutic tool. This is the reason why we started the research starting with a small selected group of subjects and we prepared a preliminary note, only based on four subjects, just to give a preliminary announce that in fact will appear on NeuroQuantology. In the present paper we give the final and conclusive results of the research realized on an articulated group of ten subjects, selected so to cover also a wide range of age.

\section{MATERIALS AND METHODS}

Ten patients, 4 males and 6 females, 28 - 62 years old, in ascertained healthy condition, were evaluated by STAI (State-trait-Anxiety Inventory) for state anxiety, by SII for stress, and BDI (Beck-depression Inventory) for depression, giving values at the boundary of the norm. Subjects had not sedentary style life and had normal weight, were tested not having other general medical, neurological and psychiatric diseases, and central nervous system acting without drugs assumption. A written informed consent was obtained before the experiment, after its approval by the local ethical committee. Subjects were instructed to abstain from caffeine, alcohol, and medication and to maintain their regular sleep-wake schedule on the 3 days before each experimental session. The experiment was conducted in accordance with the Declaration of Helsinki. The R-R tachogram recording was performed by plethysmography for five minutes in condition of absolute quiet and general comfortable condition for each subject, at rest in a quiet room with soft distant and very weak illumination and spontaneous respiration. The recorded tachogram was subsequently analyzed in frequency domain by using Fast Fourier Transform (FFT). For five minutes recorded tachogram, the FFT method enabled us to identify the three bands of interest in the frequency domain. The VLF band (Very Low Frequency) ranging from $0.003 \mathrm{~Hz}$ to $0.04 \mathrm{~Hz}$, the LF band (Low 
Frequency), ranging from $0.04 \mathrm{~Hz}$ to $0.15 \mathrm{~Hz}$, and the $\mathrm{HF}$ band (High Frequency)ranging from $0.15 \mathrm{~Hz}$ to 0.40 Hz. The physiological explanation of the VLF component of the HRV is uncertain. It seems to relate thermoregulation, rennin-angiotensin system, hormonal contributions. The LF component is correlated with sympathetic and parasympathetic modulation, whereas parasympathetic activity is considered the dominant contributor to HF power. In addition for each subject the mean $\mathrm{HR}$, the standard deviation of N-N (normal beats), and the total Variability were estimated. Soon after the initial recording of the tachogram at rest condition, the BWE section of the treatment started. Each subject was submitted to Brain Entrainment using the MP3 neuro programmer software available on line at the web site http://www.transparentcorp.com/products/np/. Being the first session of treatment, it was extended only for twenty minutes. Simultaneously his/her tachogram activity was on line monitored, recorded and elaborated.

The results were analyzed by using proper software for estimation of the HR, variability, VLF, LF and HF contributions, and total variability.

The scheme of the treatment was that we used alphastimulation employed to alleviate anxiety and stress issues. This therapeutic scheme also included the technique of the dissociation which is particularly useful for subjects with restless and trouble relaxing. The duration of each session was established to be 20 minutes being the first experience of brain entrainment for each subject.

\section{RESULTS AND DISCUSSION}

Let us start evidencing the ranges that we usually use in HRV analysis in our country. They arise from our previous experimentation performed on about one thousand normal subjects differentiated in two class age: the young subjects with age ranging from 25 to 45 years and the old subjects with age ranging from 46 to 70 years.

For the group of normal young subjects we fix the following values: Mean HR (59 - 96 bpm). Standard deviation of normal N-N beats (40 - $100 \mathrm{msec}$ ), Logarithmic value of Total Power (6.9 - 9.1 corresponding to range 992.27-8955.29 in PSD (Pwer Spectral Density)). VLF Power (5.4 - 7.1, corresponding to the range 221.40 1211.96 in PSD), LF Power (6.3 - 8.4) (544.57 - 4447.06 in PSD), HF Power (5.1 - 7.3) (164.02 - 1480.30 in PSD).

The first subject, SL, female, 30 years old, started with the following values: $89.2 \mathrm{BPM}, 36.9 \mathrm{msec}$ as standard deviation, 6.95 as Total Power (1045), 6.06 as VLF Power (430), 6.14 as LF Power(465) and 5.00 as HF Power (150) with LF/HF $=3.10$. The subject thus showed a decreased variability in HR (standard deviation) and a decreased value in the HF-vagal band. After the 20 minutes session of BWE her values were the following: 81.1 BPM, $42.3 \mathrm{msec}$ as standard deviation, (she reached the normal value), 7.44 as Total Power (1711) (she reached the normal value), 6.4 as VLF Power (602), 6.38 as LF power (591) and 6.25 (518) as HF Power (she reached a very satisfactory normal value), $\mathrm{LF} / \mathrm{HF}=1.14$. We had a reduction of about $9 \%$ in the BPM, an increase of standard deviation about $13 \%$ (increased heart rate variability), an increased 39\% in total Power variability, an increased 29\% value for the VLF Power, an increased value about $16 \%$ in LF Power and an increase about $71 \%$ in vagal HF Power. In brief, the alpha stimulation induced in the subject an improvement in the total variability and a very important improvement in parasympathetic activity.

The second subject, SR, man, 50 years old, started with the following values: 95.7 BPM, 15.9 msec as standard deviation, 5.38 as Total Power (216), 4.06 as VLF Power (58), 4.34 as LF Power (77) and 4.39 as HF Power( 81) and LF/HF $=0.95$. The subject thus showed a decreased variability in HR and decreased values in Total Power, LF and HF power. After the 20 minutes session of BWE his values were the following: 87.1 BPM, 20.1 msec as standard deviation, 5.89 as Total Power (360), 4.62 as VLF Power (102), 4.56 as LF power (96) and 5.09 as HF Power (162), LF/HF $=0.59$.

We had a reduction of about $9 \%$ in the BPM, an increase of standard deviation about 21\% (increased heart rate variability), an increase about $40 \%$ in total Power variability, an increase about $43 \%$ for the VLF, $20 \%$ for LF Power and an increase about 50\% in vagal HF Power with a final value of 5.09 almost to reach the range of the normal values for young subjects, (5.1 - 7.31). In brief, also in this case the alpha stimulation induced in the subject an improvement in the total variability and in parasympathetic activity.

The third subject, SP, female, 35 years old, started with the following values: 91.9 BPM, $28.6 \mathrm{msec}$ as standard deviation, 6.64 as Total Power (765), 4.71 as VLF Power (111), 6.15 as LF Power(471) and 5.2 as HF Power (183), LF/HF $=2.57$. The subject thus showed a decreased variability in HR but she was in rather almost accordance with the range of the normal values for Total Power, LF and HF powers. She also evidenced unbalancing in LF/HF ratio. It follows that this is a case of greater interest in order to investigate as BWE acted. After the 20 minutes session of BWE her values were the following: 83.2 BPM, $34.8 \mathrm{msec}$ as standard deviation, 7.01 as Total Power (1107), 5.48 as VLF Power (240), 6.34 as LF Power (570) and 5.69 as HF Power (297), $\mathrm{LF} / \mathrm{HF}=1.92$. We had a reduction of about $9 \%$ in the BPM, an increase of standard deviation about $28 \%$, an increase about $31 \%$ in total Power variability, an increase 
about $54 \%$ in VLF Power an increase about $17 \%$ for LF Power and an increase about 38\% vagal HF Power. In brief, also in this case the alpha stimulation induced in the subject a large improvement in the total variability and parasympathetic activity.

The fourth subject, SSc, female, 28 years old, started with the following values: 92.1 BPM, 24.9 msec as standard deviation, 6.37 as Total Power (583), 5.66 as VLF Power (288), 5.50 as LF Power (245) and 3.9 as HF Power (50) with an unbalanced ratio $\mathrm{LF} / \mathrm{HF}=4.90$. After the 20 minutes session of BWE her values were the following: 89.1 BPM, 27.9 msec as standard deviation, 6.59 as Total Power (730), 5.23 as VLF Power (187), 6.06 as LF power (431) and 4.71 as HF Power (112), LF/HF = 3.85. We had a reduction of about $3 \%$ in the BPM, an increase of standard deviation about $11 \%$, an increase in Total Power about 20\%, a decrease about 35\% for VLF, increase about $43 \%$ LF and an increase about $55 \%$ for vagal HF Power. In brief, also in this case the alpha stimulation induced in the subject a dominant improvement in the total variability and parasympathetic activity.

The fifth subject, AL, female, 30 years old, started with the following values: 95.1 BPM, $29.6 \mathrm{msec}$ as standard deviation, 6.66 as Total Power (781), 4.48 as VLF Power (89), 5.69 as LF Power (296) and 5.98 as HF Power (396) and LF/HF $=0.74$. The subject thus showed a decreased variability in HR and decreased values in Total Power, LF and HF power. After the 20 minutes session of BWE her values were the following: 89.8 BPM, $41.8 \mathrm{msec}$ as standard deviation, 7.41 as Total Power (1658), 4.29 as VLF Power (73), 6.52 as LF power (679) and 6.81 as HF Power (906), LF/HF $=0.74$.

We had a reduction of about $6 \%$ in the BPM, an increase of standard deviation about 29\% (increased heart rate variability), an increase about 53\% in total Power variability, a decrease about $18 \%$ for the VLF, $56 \%$ for LF Power and an increase about $56 \%$ in vagal HF Power with a final value of 5.09 almost to reach the range of the normal values for young subjects, (5.1 - 7.31). In brief, also in this case the alpha stimulation induced in the subject an improvement in the total variability and in parasympathetic activity.

The sixth subject, RM, man, 55 years old, started with the following values: 71.6 BPM, $24.8 \mathrm{msec}$ as standard deviation, 6.37 as Total Power (586), 5.44 as VLF Power (231), 5.70 as LF Power (298) and 4.04 as HF Power (57) with an unbalanced ratio $\mathrm{LF} / \mathrm{HF}=5.23$. After the 20 minutes session of BWE her values were the following: $70.8 \mathrm{BPM}, 39.7 \mathrm{msec}$ as standard deviation, 7.30 as Total Power (1487), 6.31 as VLF Power (549), 6.58 as LF power (723) and 5.37 as HF Power (215), LF/HF = 3.36. We had a reduction of about $1 \%$ in the BPM, an increase of standard deviation about $38 \%$, an increase in Total Po- wer about $68 \%$, a decrease about $58 \%$ for VLF, increase about $59 \%$ LF and an increase about $73 \%$ for vagal HF Power. In brief, also in this case the alpha stimulation induced in the subject a dominant improvement in the total variability and parasympathetic activity.

The seventh subject, PL, female, 60 years old, started with the following values: 57.5 BPM, 20.2 msec as standard deviation, 6.16 as Total Power (473), 4.81 as VLF Power (123), 4.82 as LF Power (124) and 5.42 as HF Power (226) with an unbalanced ratio LF/HF $=0.55$. After the 20 minutes session of BWE her values were the following: 59.3 BPM, 34.6 msec as standard deviation, 7.04 as Total Power (1141), 5.95 as VLF Power (382), 5.51 as LF power (248) and 6.24 as HF Power (511), $\mathrm{LF} / \mathrm{HF}=0.49$. We had an increase of about $3 \%$ in the BPM, an increase of standard deviation about $42 \%$, an increase in Total Power about 59\%, a decrease about $68 \%$ for VLF, increase about 50\% LF and an increase about $56 \%$ for vagal HF Power. In brief, also in this case the alpha stimulation induced in the subject a dominant improvement in the total variability and parasympathetic activity.

The eighth subject, NC, female, 62 years old, started with the following values: $75.8 \mathrm{BPM}, 13.5 \mathrm{msec}$ as standard deviation, 5.01 as Total Power (150), 4.14 as VLF Power (63), 3.74 as LF Power (42) and 3.81 as HF Power (45) with an unbalanced ratio $\mathrm{LF} / \mathrm{HF}=0.93$. After the 20 minutes session of BWE her values were the following: 74.8 BPM, $22.5 \mathrm{msec}$ as standard deviation, 6.13 as Total Power (458), 5.39 as VLF Power (220), 5.08 as LF power (161) and 4.34 as HF Power (77), $\mathrm{LF} / \mathrm{HF}=2.09$. We had a reduction of about $1 \%$ in the BPM, an increase of standard deviation about $40 \%$, an increase in Total Power about $67 \%$, a decrease about $71 \%$ for VLF, increase about $74 \%$ LF and an increase about $42 \%$ for vagal HF Power. In brief, also in this case the alpha stimulation induced in the subject a dominant improvement in the total variability and parasympathetic activity.

The ninth subject, $A B$, man, 52 years old, started with the following values: 97.3 BPM, $12.1 \mathrm{msec}$ as standard deviation, 4.74 as Total Power (115), 2.64 as VLF Power (14), 4.20 as LF Power (67) and 3.53 as HF Power (34) with an unbalanced ratio $\mathrm{LF} / \mathrm{HF}=1.97$. After the 20 minutes session of BWE her values were the following: 88.9 BPM, $16.1 \mathrm{msec}$ as standard deviation, 5.21 as Total Power (183), 3.43 as VLF Power (31), 4.56 as LF power (96) and 4.03 as HF Power (56), LF/HF = 1.71. We had a reduction of about $9 \%$ in the BPM, an increase of standard deviation about 25\%, an increase in Total Power about $37 \%$, a decrease about $55 \%$ for VLF, increase about 30\% LF and an increase about 39\% for vagal HF Power. In brief, also in this case the alpha stimulation in- 
duced in the subject a dominant improvement in the total variability and parasympathetic activity.

The tenth subject, MM, man, 58 years old, started with the following values: 92.4 BPM, $26.4 \mathrm{msec}$ as standard deviation, 6.45 as Total Power (630), 5.50 as VLF Power (244), 5.60 as LF Power (271) and 4.74 as HF Power (115) with an unbalanced ratio $\mathrm{LF} / \mathrm{HF}=2.36$. After the 20 minutes session of BWE her values were the following: $84.1 \mathrm{BPM}, 31.2 \mathrm{msec}$ as standard deviation, 6.94 as Total Power (1035), 5.86 as VLF Power (352), 5.84 as LF power (343) and 5.83 as HF Power (340), LF/HF = 1.01. We had a reduction of about $9 \%$ in the BPM, an increase of standard deviation about $15 \%$, an increase in Total Power about 39\%, a decrease about 31\% for VLF, increase about $21 \% \mathrm{LF}$ and an increase about $66 \%$ for vagal HF Power. In brief, also in this case the alpha stimulation induced in the subject a dominant improvement in the total variability and parasympathetic activity. All the increasing and decreasing values were estimated by using a coefficient of variation obtained as the difference between the greatest value and smallest value divided by the largest value.

The results are summarized in Table 1 and Figures 1(a) and (b), 2(a)-(d). As indication, we give in Figure 3 the tachogram of an examined subject before and after the BWE treatment.

\section{CONCLUSIONS}

In conclusion, we may say that in the examined subjects we had an increase in standard deviation varying from $11 \%$ to $42 \%$. An increase in Total Variability ranging from $20 \%$ to $68 \%$, an increase in VLF variability ranging from $18 \%$ to $71 \%$, (with two cases of decreasing

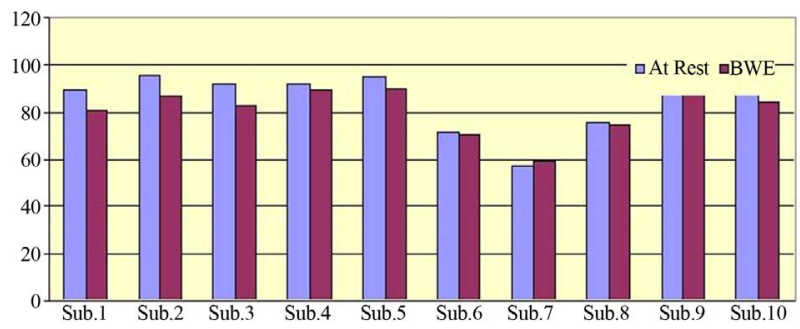

(a)

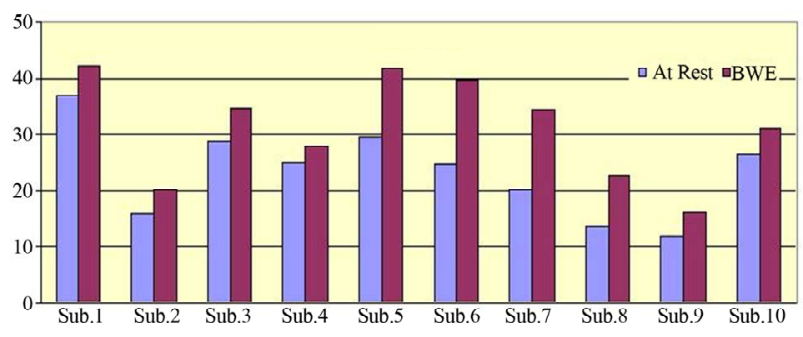

(b)

Figure 1. (a) BMP (beats/m inute); (b) SD (msec).

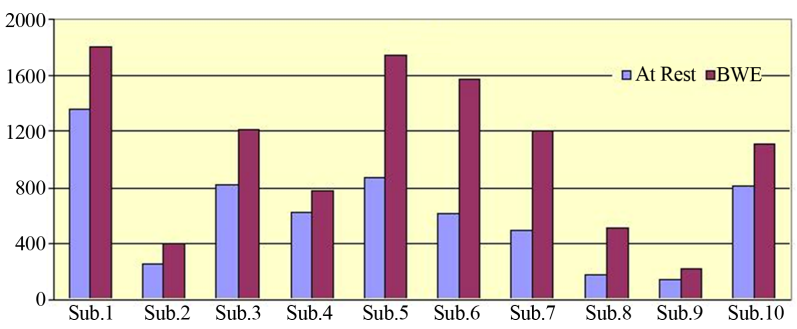

(a)

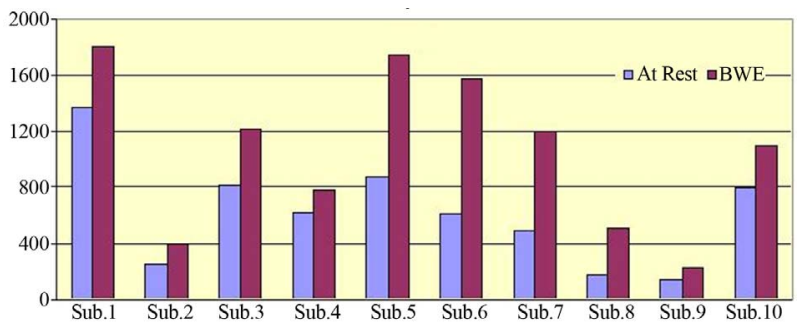

(b)

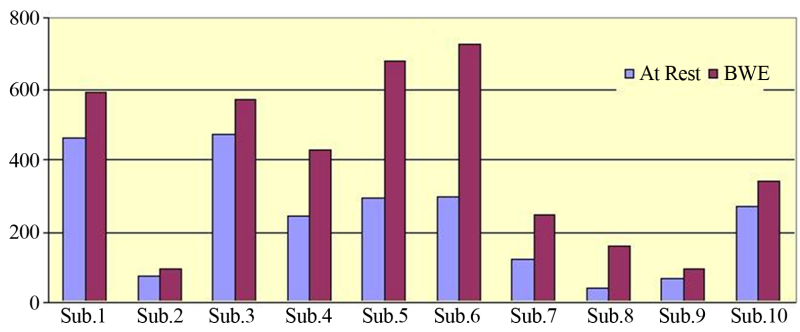

(c)

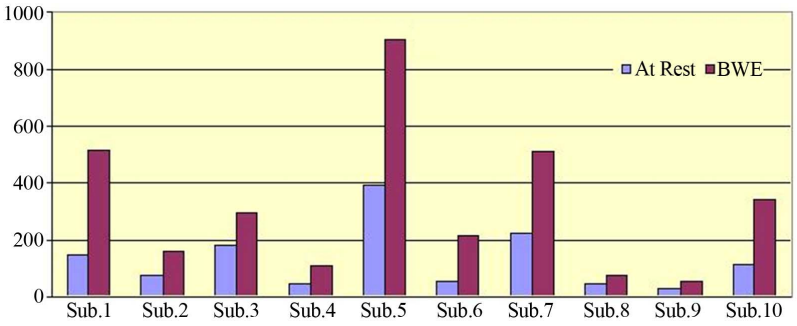

(d)

Figure 2. (a) Total Variability (T.V.); (b) Very Low Frequency (V.L.F.); (c) Low Frequency (L.F.); (d) High Frequency.

value), an increase in LF ranging from $16 \%$ to $74 \%$ and an increase in HF ranging from $38 \%$ to $73 \%$. These are very promising results.

The conclusion of the paper may be arranged in the following manner. Alpha rhythm during BWE interacts with the brain stem control in the different HRV bands taken in consideration. We could hypothesize different patterns but in our perspective we retain that the limbic system is the most appropriate to be considered.

There is still a final consideration to add. We examined a group of ten subjects reaching in this manner conclusive results. A next step is now necessary under the methodological profile. In the present paper we used the FFT since it is currently used in clinical investigations. Of course we have to outline here that the tachogram is 
Table 1. Results before and brain entrainment.

\begin{tabular}{|c|c|c|c|c|c|c|c|c|}
\hline Subject (age) & & bmp & Standard Deviation & Total Power & VLF Power & LF Power & HF Power & LF/HF \\
\hline \multirow[t]{2}{*}{ SL, F (30 years old) } & Starting & 89.2 & 36.9 & 1045 & 430 & 465 & 150 & 3.10 \\
\hline & After 20 minutes BWE & 81.1 & 42.3 & 1711 & 602 & 591 & 518 & 1.14 \\
\hline \multirow[t]{2}{*}{ SR, M (50 years old) } & Starting & 95.7 & 15.9 & 216 & 58 & 77 & 81 & 0.95 \\
\hline & After 20 minutes BWE & 87.1 & 20.1 & 360 & 102 & 96 & 162 & 0.59 \\
\hline \multirow[t]{2}{*}{ SP, F (35 years old) } & Starting & 91.9 & 28.6 & 765 & 111 & 471 & 183 & 2.57 \\
\hline & After 20 minutes BWE & 83.2 & 34.8 & 1107 & 240 & 570 & 297 & 1.92 \\
\hline \multirow[t]{2}{*}{ SSc, F (28 years old) } & Starting & 92.1 & 24.9 & 583 & 288 & 245 & 50 & 4.90 \\
\hline & After 20 minutes BWE & 89.1 & 27.9 & 730 & 187 & 431 & 112 & 3.85 \\
\hline \multirow[t]{2}{*}{ AL, F (30 years old) } & Starting & 95.1 & 29.6 & 781 & 89 & 296 & 396 & 0.75 \\
\hline & After 20 minutes BWE & 89.8 & 41.8 & 1658 & 73 & 679 & 906 & 0.75 \\
\hline \multirow[t]{2}{*}{ RM, M (55 years old) } & Starting & 71.6 & 24.8 & 586 & 231 & 298 & 57 & 5.23 \\
\hline & After 20 minutes BWE & 70.8 & 39.7 & 1487 & 549 & 723 & 215 & 3.36 \\
\hline \multirow[t]{2}{*}{ PL, F (60 years old) } & Starting & 57.5 & 20.2 & 473 & 123 & 124 & 226 & 0.55 \\
\hline & After 20 minutes BWE & 59.3 & 34.6 & 1141 & 382 & 248 & 511 & 0.49 \\
\hline \multirow[t]{2}{*}{ NC, F (62 years old) } & Starting & 75.8 & 13.5 & 150 & 63 & 42 & 45 & 0.93 \\
\hline & After 20 minutes BWE & 74.8 & 22.5 & 458 & 220 & 161 & 77 & 2.09 \\
\hline \multirow[t]{2}{*}{ AB, M (52 years old) } & Starting & 97.3 & 12.1 & 115 & 14 & 67 & 34 & 1.97 \\
\hline & After 20 minutes BWE & 88.9 & 16.1 & 183 & 31 & 96 & 56 & 1.71 \\
\hline \multirow[t]{2}{*}{ MM, M (58 years old) } & Starting & 92.4 & 26.4 & 630 & 244 & 271 & 115 & 2.36 \\
\hline & After 20 minutes BWE & 84.1 & 31.2 & 1035 & 352 & 343 & 340 & 1.01 \\
\hline
\end{tabular}

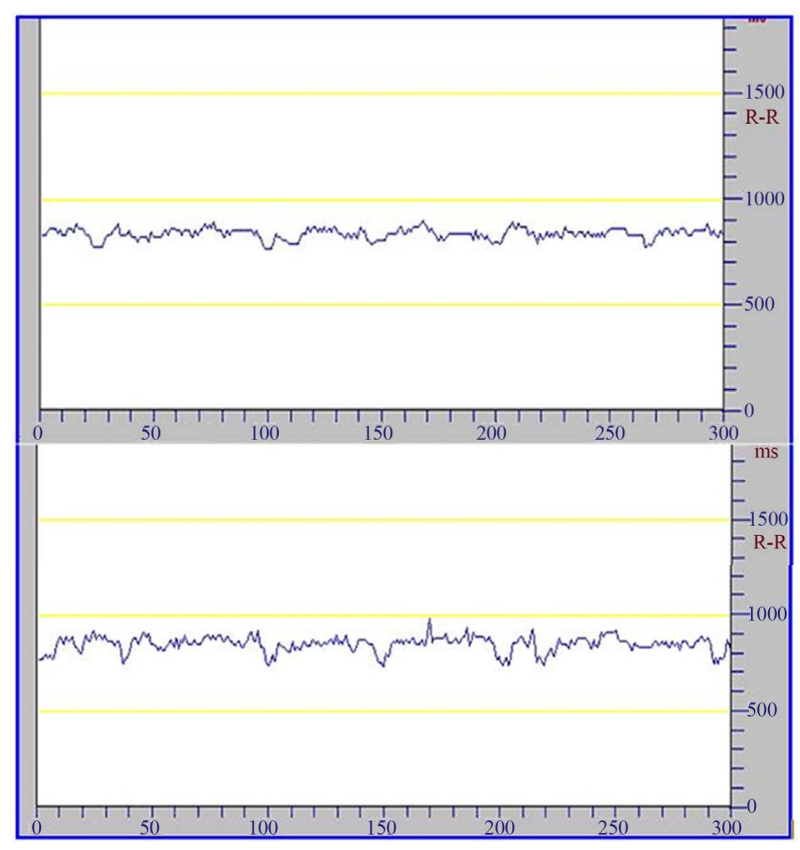

Figure 3. Initial tachogram and tachogram after brain entrainment. in itself a non linear, non stationary and non periodic signal whose complete investigation requires to add to the previous FFT linear analysis, the application of non linear methodologies. This is the following step that we have in progress and it will enable us to introduce further appropriate new indexes for more appropriate quantification of the treatment.

\section{REFERENCES}

[1] Huang, T.L. and Charyton, C. (2008) A comprehensive review of the psychological effects of brainwave entrainment. Alternative Therapies, 14, 38-49.

[2] Conte, E., Federici, A. and Zbilut, J.P. (2009) A new method based on fractal variance function for analysis and quantification of sympathetic and vagal activity in variability of $R-R$ time series in ECG signals. Chaos, Solitons and Fractals, 41, 1416-1426. http://dx.doi.org/10.1016/j.chaos.2008.05.025

[3] Conte, E., Khrennikov, A., Federici, A. and Zbilut, J.P. (2009) Fractal fluctuations and quantum-like chaos in the brain by analysis of variability of brain waves: A new method based on a fractal variance function and random 
matrix theory: A link with El Naschie fractal Cantorian space-time and V. Weiss and $\mathrm{H}$. Weiss golden ratio in brain. Chaos, Solitons and Fractals, 41, 2790-2800. http://dx.doi.org/10.1016/j.chaos.2008.10.016

[4] Conte, E., Todarello, O., Conte, S., Mendolicchio, L. and Federici, A. (2010) Methods and applications of nonlinear analysis in neurology and psycho-physiology. Journal of Consciousness Exploration \& Research, 1, 1070-1138.

[5] Conte, E., Pieralice, M., Laterza, V., Losurdo, A., Santacroce, N., Conte, S., Federici, A. and Giuliani, A. (2012) Traditional and a new methodology for analysis of heart rate variability: A review by physiological and clinical experimental results. International Journal of Research and Reviews in Applied Sciences, 13, 206-293.

[6] Sandercock, G.R., Bromley, P.D. and Brodie, D.A. (2005) The reliability of short-term measurements of heart rate variability. International Journal of Cardiology, 103, 238-247. http://dx.doi.org/10.1016/j.ijcard.2004.09.013

[7] Vaschillo, E.G., Vaschillo, B. and Lehrer, P.M. (2006) Characteristics of resonance in heart rate variability stimulated by biofeedback. Applied Psychophysiology and Biofeedback, 31, 129-142. http://dx.doi.org/10.1007/s10484-006-9009-3 